\title{
Area Spectral Efficiency Performance Comparison of Downlink Fractional Frequency Reuse Schemes for MIMO Heterogeneous Networks
}

\author{
Sherief Hashima*, Osamu Muta ${ }^{\dagger}$, Masoud Alghonimey ${ }^{\S}$, Hossam Shalaby*, Hiroshi Frukawa ${ }^{\dagger}$, Said Elnoubi ${ }^{\S}$, \\ and Imbaby Mahmoud ${ }^{\mathbb{T}}$ \\ *Egypt-Japan University of Science and Technology (E-JUST), \\ Alexandria, Egypt \\ Email: sherif.mostafa@ejust.edu.eg; shalaby@ieee.org \\ ${ }^{\dagger}$ Kyushu University \\ 744 Motooka, Nishi-ku, Fukuoka-shi, Fukuoka-ken, 819-0395 Japan \\ Email: muta@ait.kyushu-u.ac.jp;furuhiro@ait.kyushu-u.ac.jp; \\ Alexandria University \\ 21544 Alexandria, Egypt \\ Email:Alghoniemy@alexu.edu.eg;saidelnoubi@yahoo.com \\ 『Engineering Department, Egyptian Atomic Energy Authority, \\ Inshas, Egypt \\ Email:imbabyisma@yahoo.com
}

\begin{abstract}
In cellular networks, fractional frequency reuse (FFR) is an effective inter-cell interference mitigation technique to achieve significant coverage improvement for cell edge users. Area Spectral Efficiency (ASE) is an important and vital factor to be improved in cellular networks and is defined as the number of transmitted information bits per unit area per unit time per unit bandwidth (i.e., bps $/ \mathrm{Hz} / \mathrm{m}^{2}$ ). In this paper, we compare ASE of different downlink FFR schemes in MIMO Heterogeneous cellular networks ( MIMO HetNets), where the base station (BS) locations are modeled using Poission Point Process (PPP). Stochastic PPP is widely used in the design and analysis of signal processing algorithms especially for communication systems. ASEs are evaluated based on coverage probability functions for Strict FFR and Soft Frequency Reuse (SFR) in MIMO HetNets for both closed and open access cases. In the same time we illustrate not only the role but also the benefits of utilizing PPP in modeling the BS locations for analytical evaluation of two main types of FFR deployments. Numerical results show that, for SFR in both open and closed access cases, it is better to use MIMO HetNets than other techniques due to large interference resulted from utilizing the whole bandwidth inside the cell. Also, in SFR case, ASE is directly proportional to the power control factor $\beta_{k}$. For Strict FFR MIMO HetNet open access case, SDMA with large numbers of antenna provides higher ASE at the expense of system complexity. For better ASE in MIMO HetNet system, it is recommended to use adaptive technique between strict FFR MIMO and MU-MIMO in terms of target threshold value of signal-to-interference ratio (SIR).
\end{abstract}

Keywords-Area Spectral Efficiency (ASE), Multiple Input Multiple Output (MIMO), Heterogeneous network (HetNet), Fractional frequency reuse (FFR), Frequency reuse factor $(F R F)$, and Soft frequency reuse (SFR).

\section{INTRODUCTION}

Nowadays wireless data traffic is increasing exponentially, leading to a new generation of devices (smart phones, netbooks, etc.). In the same time, service migration from voice centric to data centric, leads more users operate indoors, which needs increased link budget and coverage extension to serve these users. Heterogeneous networks (HetNets) is the suitable solution deployed by Third generation Partnership Project (3GPP) Long Term Evolution-Advanced (LTE-A) as an efficient way not only to increase system capacity, but also to enhance network coverage. Nowadays Cellular networks are randomly deployed to handle increasing data requirements due to a lot of hungry data applications such as video streaming and real time video calls. Two main strategies were utilized to support such higher data rates, they are: i) low power nodes deployment such as femtocells, and ii) increase number of BSs antennas by utilizing multiple antenna techniques such as Space Division Multiple Access (SDMA), and antenna beam forming $(\mathrm{BF})$. The migration of cellular networks from old ordinary deployments towards nearly random deployments indicates that old assumptions of BS locations e.g. deterministic models, are no longer applicable. A more suitable method is to model HetNets based on utilizing random spatial models, where a Poisson point process (PPP) is used to model the BS locations [1].

Fractional Frequency Reuse (FFR) is an efficient and important ICIC technique in OFDMA based wireless network [2]. The main idea of FFR is to divide the cell bandwidth so that (i) Cell Edge Users (CEUs) in neighboring cells does not interfere with each other, and (ii) it mitigates the interference created by interior users, while (iii) utilizing the available spectrum more efficiently than traditional frequency reuse. FFR utilization in cellular systems leads to a trade off between network throughput and spectral efficiency.

There are two common FFR techniques: Strict FFR and Soft Frequency Reuse(SFR). Although FFR is applicable to both the Uplink (UL) and Downlink (DL), this work focuses on analysis in downlink of cellular networks, where equal transmission power is assumed in each Base Station (BS) without transmit power control unlike the UL.

1) Strict FFR: the left sub-figure in Fig.1 illustrates potential strict FFR frequency allocations with $D=3$. It is a modification of ordinary frequency reuse utilized extensively in cellular networks [3]. According to the user location in the cell if it is Cell Center User (CCU) or CEU, the bandwidth is allocated to that user in a different way. For CCU it uses one common sub-band of frequencies, while for CEUs the bandwidth is divided across the cells based on a reuse factor of $D$. Hence, Strict FFR requires a total of $(D+1)$ subbands. CCUs do not share any spectrum with CEUs, leading to interference mitigation especially for cell edge users.

2) Soft Frequency Reuse : it employs the same edge partitioning method as Strict FFR but CCUs are allowed to 
share the bandwidth with neighboring cells with lower power levels for transmission than the CEUs [8],[9]. The right subfigure in Fig.1 illustrates the power and frequency allocation for SFR. The whole bandwidth is equally divided to $D$ subbands as shown in Fig.1, where $D$ denotes the frequency reuse factor FRF. Although SFR is more spectral efficient than Strict FFR, it introduces larger interference to both CEUs, and CCU. This is because the cell edge users share the same sub band with close cell interior users. Hence power control is applied in SFR, where cell edge users downlinks are transmitted with higher power than cell interior users.

In [5], the authors analytically evaluated two main types of FFR deployments: strict FFR, and Soft Frequency Reuse (SFR) [7], [8], and [10] utilized for homogeneous networks. They modeled the BSs locations as homogeneous PPP. Also,they compared their results with not only standard grid model but also with an actual urban deployment model to validate their model. A closed form expression for not only the coverage probability but also the average data rate of both strict FFR, and SFR were obtained. The same authors discussed the trade off problem between strict FFR, and SFR regarding QOS and overall capacity in [6]. In [11], the same authors derived the same model but for Heterogeneous networks that employ strict FFR and SFR techniques. They derived a closed form expression for both coverage probability, and average data rate in both closed and open access cases. The derived expressions are tractable and very helpful for system design.

Sufficient modeling of single antenna HetNets was started in [17], and [18] and completed in [16]. The authors provided a new tractable model for K-tier downlink HetNets and gave a closed form expressions for the most performance metrics like coverage probability and data rate based on random spatial model in [16]. They considered homogeneous user distribution in their analysis with all BSs transmitting at all times. In [19], the authors started to model MIMO HetNets. In [20], a closed form expression for the downlink coverage probability of MIMO HetNets assuming $\mathrm{K}$ tiers of BSs which may have various transmit power, threshold Signal to Interference Ratio, user (deployment) density, number of transmit antennas, and multi-antenna technique. The authors also derived an upper bound for the coverage probability by making use of useful tools from stochastic geometry. In [21], the same analysis was performed for MIMO heterogeneous networks that utilize Strict FFR and SFR. A closed form expressions for the coverage probability of MIMO FFR Hetnet was obtained for both closed and open access cases. The derived coverage probabilities of this paper are used in our paper to numerically calculate the ASE of different FFR MIMO HetNet schemes.

In this paper, we compare various FFR schemes in SISO and MIMO HetNet in view point of Area Spectral Efficiency (ASE). ASE is an important metric for comparison between different HetNet systems, and defined as the number of bits transmitted per unit area per unit time per unit bandwidth (i.e., bps $/ \mathrm{Hz} / \mathrm{m}^{2}$ ). This paper uses ASE to clarify the effect of FFR on wireless systems especially in HetNets. The rest of this paper is organized as follows: Section II describes the general system model for analysis and previews the concept of ASE. Coverage probability expressions of SISO, MU-MIMO, strict FFR, and SFR MIMO HetNets closed access case are surveyed in Sec. III. Section IV discusses open access case based on open access coverage probability expressions. Section V provides the corresponding simulation results. Finally, section VI concludes the work.

\section{SySTEM MODEL}

Consider both SISO and MIMO HetNets with $K$ different tiers of Base stations (BSs). BSs at different tiers have different transmit powers $P_{k}$, different user densities $\lambda_{k}$, different threshold values $T_{k}$, and different number of

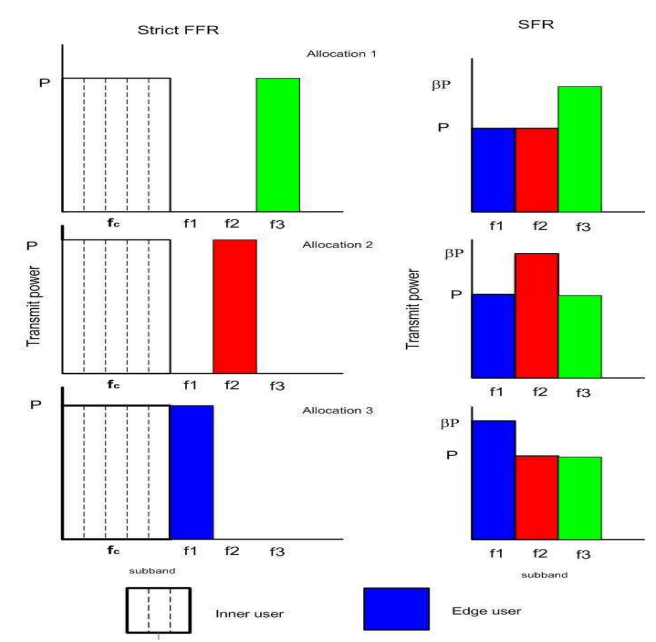

Fig. 1: Strict FFR and SFR sub band and transmit power allocation with $D=3$.

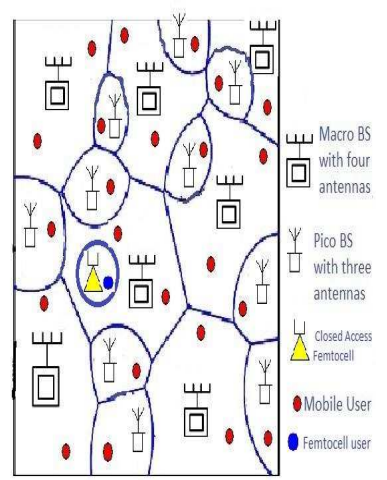

(a) Closed Access

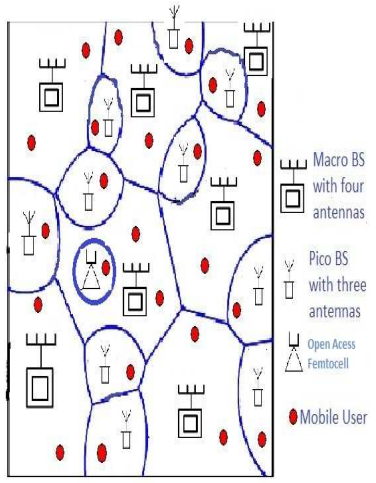

(b) Open Access
Fig. 2: An illustration of a possible two tier MIMO HetNet configuration with four antenna macro BSs, and three antenna pico BSs in both closed and open access cases.

antennas $M_{k}$. In this model we will put into consideration SIR not SINR because HetNets are a typical interference limited network [11]. Each BS location at $k_{t h}$ tier is modeled as independent PPP with density $\lambda_{k}$ [12]. PPP model assumption has been validated for large cells network by observation in [13], and theoritically under channel randomness in [14]. Putting into consideration not loosing generality, our focus in downlink analysis is on a typical single antenna mobile user located at the origin. The mobile user can access to just one tier and to the closest BS of the tier at a time. $x_{k}$ is the distance between the mobile user and its nearest serving BS. $h_{k x}$ is the direct link channel gain from that mobile user and its serving $k_{t h}$ tier BS. $g_{i y}$ is the indirect (interfering) link channel gain from $j_{t h}$ tier BS located at distance $y_{k}$ from that mobile user. Both $h_{k x}$ and $g_{i y}$ follow gamma distribution [15] such that $h_{k x} \sim \Gamma\left(\Delta_{k}, 1\right)$, and $g_{i y} \sim \Gamma\left(\psi_{j}, 1\right)$, where $\Delta_{k}$, and $\psi_{j}$ are positive integers and depend on the adopted multi antenna technique. Table.I previews different multi-antenna cases where $M_{k}$ is an integer that indicates the number of antennas in each tier.

The received power of that typical mobile user at the origin 
TABLE I: multi-antenna technique cases using $\psi_{k}$, and $\Delta_{k}$.

\begin{tabular}{|l|l|l|}
\hline \hline Multi-antenna technique & $\psi_{k}$ & $\Delta_{k}$ \\
\hline Space Division Multiple Access (SDMA) & $\psi_{k}=M_{k}$ & $\Delta_{k}=1$ \\
\hline $\begin{array}{l}\text { Multi-Input Single Output (MISO), } \\
\text { i.e, Single User beam forming (SU-BF) }\end{array}$ & $\psi_{k}=1$ & $\Delta_{k}=M_{k}$ \\
\hline Single-Input Single-output (SISO) & $\psi_{k}=1$ & $\Delta_{k}=1$ \\
\hline \hline
\end{tabular}

from the serving BS is given as $P_{r}=P_{k} h_{k x_{k}} x_{k}^{-\alpha}$, where $\alpha$ is the path loss exponent. The received SIR of that typical user is given by

$$
\operatorname{SIR}\left(x_{k}\right)=\frac{P_{k} h_{k x_{k}} x_{k}^{-\alpha}}{\sum_{j \in K} \sum_{y \in \phi_{k}} P_{j} g_{j y} y_{k}^{-\alpha}}
$$

\section{A. Area Spectral Efficiency}

The mostly used parameters for comparing various system configuration and transmission techniques is the coverage probability and the rate per user. However, these two parameters does not directly account the fact that some techniques like Space Division Multtiple Access (SDMA) serve large number of users than the others, such as Single User Beam Forming (SU-BF) case which is MISO multi antenna technique, and may result in higher sum data rate. We use ASE to account this fact, as it gives the number of bits transmitted per unit area per unit time per unit bandwidth [20]. ASE for multi-tier can be given as:

$$
A S E=\sum_{k \in K} \psi_{k} \lambda_{k} \log _{2}\left(1+T_{k}\right) P_{c}^{(k)}
$$

where $P_{c}^{(k)}$ is the per tier coverage probability, and $T_{k}$ is the per tier target threshold. For analytical comparison simplicity, we will limit our comparison to $P_{c}^{(k)}=P_{c}$ for all tiers. We also assume equal number of antennas per BS and target threshold for all tiers (i.e. $T_{k}=T$ ). ASE under these assumptions is given by:

$$
A S E=P_{c} \log _{2}(1+T) \sum_{k \in K} \psi_{k} \lambda_{k}
$$

\section{ClOSED ACCESS ANALYSIS}

In this section, we evaluate the downlink different ASEs (i.e. SISO, MIMO, strict FFR MIMO, and SFR MIMO) of multi-tier network with closed access between the tiers. For example, a three tiers network with macrocells and underlaid femtocells. A typical mobile user served by a macrocell may be in range of a closed access femtocell, but unable to connect to that femtocell, resulting more cross tier interference. The coverage probability equation of that mobile user is utilized to numerically calculate ASE at different MIMO techniques. In our analysis, the mobile user is under coverage of $k_{t h}$ tier if its SIR from the closest BS of $k_{t h}$ tier is larger than a threshold $T_{k}$. The coverage probability is the complementary cumulative distribution (CCDF) function of SIR, i.e. $P_{c}=P\left(S I R_{k} \succ T_{k}\right)$. ASE is numerically calculated by substituting coverage probability equations and other parameters to (3).

\section{A. Strict FFR, MIMO HetNet, Closed access}

Here we are interested in the CEU's ASE of a CEU. Hence the coverage probability will be a conditional function defined as

$$
P c_{\text {strictFFR,cl }}=P\left(S I R_{\text {edge }} \succ T / S I R_{k} \prec T_{k}\right)
$$

The coverage probability of edge user, closed access, Strict FFR, MIMO HetNet is obtained in theorem 1 in [21], as following:

$$
P_{F F R, c l}=\frac{\int_{0}^{\infty} \rho(k, \lambda) \cdot f_{1}\left(s_{1 x_{k}}^{\prime}\left(1-f_{1}^{\prime}\left(s_{1 x_{k}}\right)\right)\right) d x_{k}}{\int_{0}^{\infty} \rho(k, \lambda)\left(1-f_{1}^{\prime}\left(s_{1 x_{k}}\right)\right) d x_{k}}
$$

where $s_{1 x_{k}}^{\prime}=\frac{T x_{k}^{\alpha}}{P_{k} D_{k}}, s_{1 x_{k}}=\frac{T_{k} x_{k}^{\alpha}}{P_{k}}$,

$$
\rho(k, \lambda)=2 \pi x_{k} \lambda_{k} \exp \left(-\pi x_{k}^{2} \lambda_{k}\right)
$$

$$
f_{1}(x)=\sum_{i=0}^{\Delta_{k}-1} \frac{1}{i !}\left[(-x)^{i} \frac{\delta^{i}}{\delta(x)^{i}} \exp \left(-x^{\frac{2}{\alpha}} \lambda_{k} P_{k}^{\frac{2}{\alpha}} C\left(\alpha, \psi_{k}\right)\right)\right]
$$

$$
\begin{gathered}
f_{1}^{\prime}(x)=\sum_{i=0}^{\Delta_{k}-1} \frac{1}{i !}\left[(-x)^{i} \frac{\delta^{i}}{\delta(x)^{i}} \exp \left(-x^{\frac{2}{\alpha}} \sum_{j=1}^{K} \lambda_{k} P_{k}^{\frac{2}{\alpha}} C\left(\alpha, \psi_{k}\right)\right)\right] \\
C(\alpha, M)=\frac{2 \pi}{\alpha} \sum_{m=1}^{M}\left(\begin{array}{c}
M \\
m
\end{array}\right) B\left(M-m+\frac{2}{\alpha}, m-\frac{2}{\alpha}\right) \\
B(x, y)=\int_{0}^{1} t^{x-1}(1-t)^{y-1} d t \text { is the euler beta function. }
\end{gathered}
$$

\section{B. SFR, MIMO HetNet, Closed access}

In case of SFR, $\beta$ ranges from 0 to $20 \mathrm{~dB}$ [4] and it is large than 1. For $K$ tiers HetNet that employs SFR, the interference of the cell edge user will equal $\sum_{k=1}^{K} \eta_{k} I_{k}$, where $I_{k}=\sum_{y \in \phi_{k}} P_{k} g_{k y} y_{k}^{-\alpha}$ and $\eta_{k}=\left(D_{k}-1+\beta_{k}\right) / D_{k}$ merges the interior and edge down link interference into one effective interference term [11]. In a similar method strict FFR, but with considering SFR power control factor $\beta$, the coverage probability of $k^{\text {th }}$ edge user, SFR, closed access, MIMO channel in the MIMO HetNets is derived in theorem 2 [11], and its equation is given as

$$
\begin{gathered}
P_{S F R, c l}=\frac{\int_{0}^{\infty} \rho(k, \lambda) \cdot f_{2}\left(s_{2 x_{k}}^{\prime}\left(1-f_{2}^{\prime}\left(s_{2 x_{k}}\right)\right)\right) d x_{k}}{\int_{0}^{\infty} \rho(k, \lambda)\left(1-f_{2}^{\prime}\left(s_{2 x_{k}}\right)\right)} d x_{k} \text { (10) } \\
s_{2 x_{k}}^{\prime}=\frac{T x_{k}^{\alpha}}{\beta_{k} P_{k}}, s_{2 x_{k}}=\frac{T_{k} x_{k}^{\alpha}}{P_{k}}, \text { and } \\
f_{2}(x)=\sum_{i=0}^{\Delta_{k}-1} \frac{1}{i !}\left[(-x)^{i} \frac{\delta^{i}}{\delta(x)^{i}} \exp \left((-x)^{\frac{2}{\alpha}} \sum_{j=1}^{K} \lambda_{j}\left(\eta_{j} p_{j}\right)^{\frac{2}{\alpha}} C\left(\alpha, \psi_{j}\right)\right)\right.
\end{gathered}
$$

\section{SISO HEtNet, Closed access}

The coverage probability of closed access SISO HEtNet is derived in [11] collary 3 and given in (12). An important notice is that, closed access has lower coverage than open access by a factor $\frac{\sum_{i \in B} \lambda_{i}}{\sum_{i=1}^{K} \lambda_{i}}$, where $B$ is the subset of tiers that a mobile user can connect to in closed access scenario.

$$
p_{S I S O, c l}=\frac{\pi}{C(\alpha)} \frac{\sum_{i \in B} \lambda_{i} P_{i}^{\frac{2}{\alpha}} T_{k}^{\frac{-2}{\alpha}}}{\sum_{i=1}^{K} \lambda_{i} P_{i}^{\frac{2}{\alpha}}}=\frac{\pi}{C(\alpha) T^{\frac{2}{\alpha}}} \frac{\sum_{i \in \beta} \lambda_{i}}{\sum_{i=1}^{K} \lambda_{i}}
$$




\section{OPEN ACCESS ANALYSIS}

In this section, we consider only two tiers analysis for complexity reduction without loss of generality. In open access case, there are no closed access femtocells, hence a typical mobile user served by macrocell and in the same time in a femtocell range. This user can be easily connected to that femtocell if he has lower SIR from the macrocell. The SIR of first and second tiers are defined as $S I R_{1}$, and $S I R_{2}$. Their target thresholds are $T_{1}$, and $T_{2}$, respectively. The typical user is CEU only when $S I R_{1} \prec T_{1}$, and $S I R_{2} \prec T_{2}$. The CEU coverage probability will be also a conditional one, denoted as

$$
P c_{o p}=P\left(S I R_{e d g e} \succ T / S I R_{1} \prec T_{1}, S I R_{2} \prec T_{2}\right)
$$

In this section, ASE can be given by substituting each coverage probability to $P_{c}$ in (3). With the knowledge of other parameters like number of tiers $k$, number of antennas in each tier $\psi_{k}$ and deployment density of each tier $\lambda_{k}$ we can easily got the ASE of each scheme.

\section{A. MU-MIMO HEtNet, Open Access}

The coverage probability in a K-tier MU MIMO HetNet with each $k^{t h}$ tier BS serving $M_{k}$ users [20] is given by

$$
p_{M U-M I M O}=\pi \frac{\sum_{k \in K} \lambda_{k} P_{k}^{\frac{2}{\alpha}} T_{k}^{\frac{-2}{\alpha}}}{\sum_{j \in K} \lambda_{j} P_{j}^{\frac{2}{\alpha}} C\left(\alpha, M_{j}\right)}
$$

\section{B. SISO HEtNet, Open Access}

Like closed access case, the coverage probability of SISO HetNet at open access [11] is given as follow

$$
p_{S I S O, O P}=\frac{\pi}{C(\alpha)} \frac{\sum_{i=1}^{K} \lambda_{i} P_{i}^{\frac{2}{\alpha}} T_{k}^{\frac{-2}{\alpha}}}{\sum_{i=1}^{K} \lambda_{i} P_{i}^{\frac{2}{\alpha}}}=\frac{\pi}{C(\alpha) T^{\frac{2}{\alpha}}}
$$

\section{Strict FFR, MIMO HetNet, Open Access}

The coverage probability of first tier cell-edge user in MIMO HetNets with strict FFR, open access case [21] is given by

$p_{F F R, o p}=\frac{\int_{0}^{\infty} \int_{0}^{\infty} \rho(1, \lambda) \cdot \rho(2, \lambda) f\left(s s^{\prime}\right)\left(f_{3 d}\left(s, s^{\prime}, s s\right)\right) d x_{1} d x_{2}}{\int_{0}^{\infty} \int_{0}^{\infty} \rho(1, \lambda) \cdot \rho(2, \lambda) f_{3 d}\left(s, s^{\prime}, s s\right) d x_{1} d x_{2}}$

where $s=\frac{T_{1} x_{1}^{\alpha}}{P_{1}}, s^{\prime}=\frac{x_{1}^{\alpha}}{P_{1}}, s s=\frac{P_{2} x_{2}^{-\alpha}}{T_{2}}, s s^{\prime}=\frac{T x_{1}^{\alpha}}{P_{1} D_{1}}, \rho=$ $\rho(1, \lambda) \cdot \rho(2, \lambda)$

$$
\begin{gathered}
f\left(s s^{\prime}\right)=\left(\sum_{i=0}^{\Delta_{1}-1} \frac{1}{i !}\left[\left(-s s^{\prime}\right)^{i} \frac{\delta^{i}}{\delta\left(s s^{\prime}\right)^{i}} f_{3}\left(s s^{\prime}, 1\right)\right)\right. \\
f_{3}(x, k)=\exp \left(-x^{\frac{2}{\alpha}} \lambda_{k} P_{k}^{\frac{2}{\alpha}} C\left(\alpha, \psi_{k}\right)\right)
\end{gathered}
$$

$$
\begin{aligned}
& f_{3 d}\left(s, s^{\prime}, s s\right)= \\
& -\sum_{i=0}^{\Delta_{1}-1} \frac{1}{i !}\left[(-s)^{i} \frac{\delta^{i}}{\delta(s)^{i}}\left(f_{3}(s, 1) \cdot f_{3}(s, 2) \cdot \frac{1}{\left(1+s P_{2} x_{2}^{-\alpha}\right)^{\Delta_{2}}}\right)\right. \\
& +f_{3 d-2}\left(s^{\prime}, s_{2}^{\prime}\right)
\end{aligned}
$$

where $n=\Delta_{2}-1$, and $f_{3 d-2}\left(s^{\prime}, s_{2}^{\prime}\right)$ equation is

$$
\begin{aligned}
& f_{3 d-2}\left(s^{\prime}, s_{2}^{\prime}\right) \\
& =\sum_{i=0}^{\Delta_{1}-1} \frac{1}{i !} \frac{\delta^{i}}{\delta\left(s^{\prime}\right)^{i}} \frac{n !}{\Gamma\left(\Delta_{2}\right)}+\frac{1}{\Gamma\left(\Delta_{2}\right)} \sum_{m=0}^{n} \frac{n !}{(n-m) !} \\
& \quad \times\left(s_{2}^{\prime}\right)^{m-n}\left(\frac{1}{\left(1+s^{\prime} s_{2}^{\prime}\right)^{m+1}}-1\right)(-1)^{n-m} \\
& \left.\quad \times \frac{\delta^{n-m}}{\delta\left(\frac{1}{s_{2}^{\prime}}\right)^{n-m}}\left(f_{3}\left(\frac{1}{s_{2}^{\prime}}, 1\right)\right) f_{3}\left(\frac{1}{s_{2}^{\prime}}, 2\right)\right)
\end{aligned}
$$

\section{SFR, MIMO HetNet, Open Access}

The coverage probability of the first tier CEU in MIMO HetNets with SFR, and closed access [21] is given by

$p_{S F R, o p}=\frac{\int_{0}^{\infty} \int_{0}^{\infty} \rho(1, \lambda) \rho(2, \lambda)\left(f_{4}(s)\right) \cdot\left(f_{3 d}\left(s, s^{\prime}, s_{2}^{\prime}\right)\right) d x_{1} d x_{2}}{\int_{0}^{\infty} \int_{0}^{\infty} \rho(1, \lambda) \rho(2, \lambda) \cdot f_{3 d}\left(s, s^{\prime}, s_{2}^{\prime}\right) d x_{1} d x_{2}}$

where $s=\frac{T x_{1}^{\alpha}}{\beta_{1} P_{1}}$

$$
\begin{aligned}
f_{4}(s)= & \sum_{i=0}^{\Delta_{1}-1} \frac{1}{i !}\left[( - s ) ^ { i } \frac { \delta ^ { i } } { \delta ( s ) ^ { i } } \left(\exp \left(-s^{\frac{2}{\alpha}} \lambda_{1}\left(\eta_{1} P_{1}\right)^{\frac{2}{\alpha}} C\left(\alpha, \psi_{1}\right)\right)\right.\right. \\
& \times\left(\exp \left(-s^{\frac{2}{\alpha}} \lambda_{2}\left(\eta_{2} P_{2}\right)^{\frac{2}{\alpha}} C\left(\alpha, \psi_{2}\right)\right)\right]
\end{aligned}
$$

\section{NumERicAl RESUlts}

We propose three tiers interference limited for closed access case and two tiers for open access. Simulation parameters are as follows: $P_{k}=[20,5,1] \mathrm{W}$ (i.e. power of first tier $P_{1}=20 \mathrm{~W}$, second tier $P_{2}=5 \mathrm{~W}$, and third tier $\left.P_{3}=1 \mathrm{~W}\right), \lambda_{k}=[\lambda, 3 \lambda, 9 \lambda]$, $\lambda=24, T_{k}=[3,3,3] \mathrm{dB}$, path loss exponent $\alpha=4$, $D_{k}=[3,3,3]$. Open access parameters are the first two numbers of closed access parameters. ASE is plotted for FFR MIMO HetNet with its three different multi antenna schemes discussed previously in table.I. In addition, ASE curves for the MIMO only HetNet with both $\mathrm{M}=2$, and 8 and for SISO HetNet cases is plotted with FFR curves to show how much FFR schemes affect on the system.

\section{A. Closed Access Numerical Results}

Fig.3 shows the area spectral efficiency of the first tier CEU for both SISO HetNet, MU-MIMO HetNet with $m=2$, and 8 , and strict FFR with different multi antenna techniques (SISO, SDMA, and SU-BF) with different number of antennas,i.e., $M_{k}=1,2$, and 8 . ASE is calculated numerically and plotted by substituting the coverage probability, and other parameters of each scheme in (3). It is obvious from the curve that MU-MIMO HetNet ASE dominates at low SIR values, while strict FFR SDMA HetNets dominates at high threshold values. Strict FFR SU-BF ASE is lower than its similar of SDMA, and MU-MIMO. This is because strict FFR SDMA has small larger coverage than MU-MIMO. At low $T$ values, ASE depends totally on the rate more than the coverage hence MUMIMO dominates, but at large $T$ values the larger coverage dominates which is strict FFR. The case of SISO strict FFR has better ASE performance than SISO HetNet but both have the lowest ASE performance.

Fig.4 previews the comparison of SISO, MIMO, and SISO, SU-BF, and SDMA SFR in a three tier HetNet in terms of their ASE. The three Tiers are assumed to follow the same transmission technique and $\beta=[16,16,16]$ for SFR MIMO HetNet case. MIMO, and SISO only HetNets ASE outperforms 


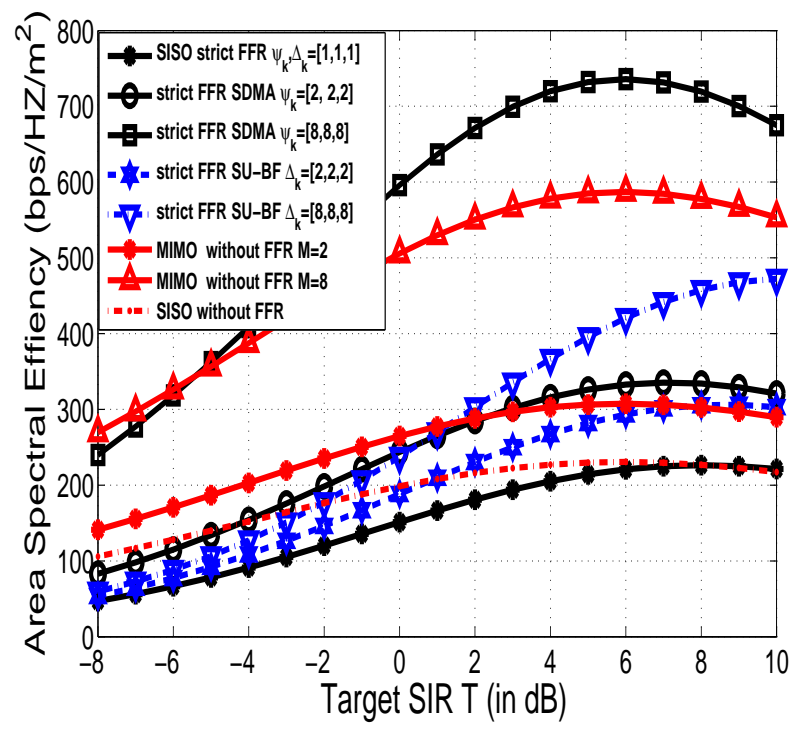

Fig. 3: Closed Access Strict FFR DL ASE for first tier cell edge user in HetNet.

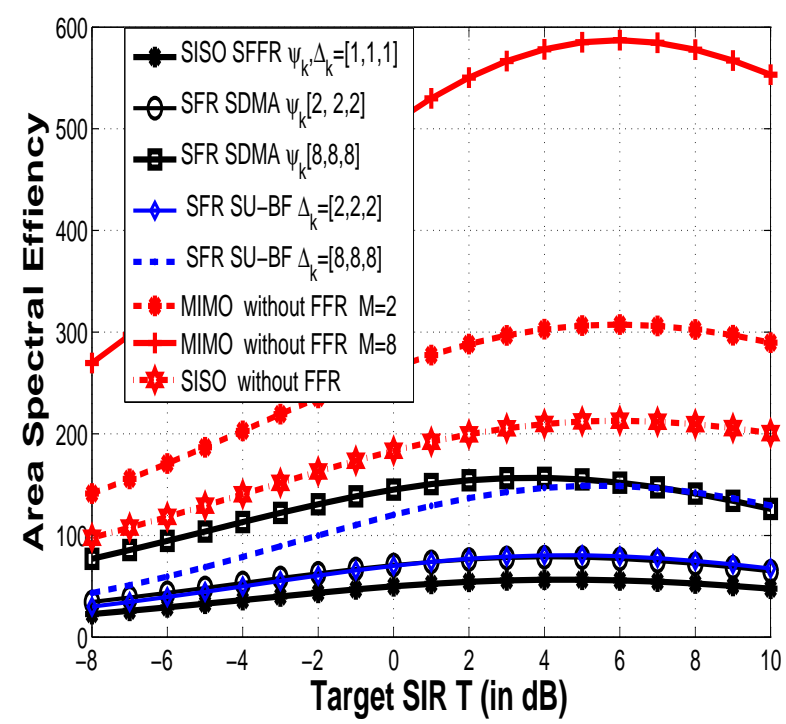

Fig. 4: Closed Access SFR DL ASE for first tier cell edge user at $\beta_{k}=[16,16,16]$ in HetNet.

ASE of three different schemes of SFR MIMO HetNets. This is because of the large interference resulted from using the whole bandwidth inside the cell in case of SFR resulting in lower coverage probability. Both SU-BF and SDMA have close performance to each other in case of SFR, closed access. Fig.5 repeats the analysis but for $\beta_{k}=[2,2,2]$. The reduction of $\beta$ leads to reduction of coverage probability in case of SFR, Hence reduction of ASE curves of SFR SISO, SU-BF, and SDMA.

\section{B. Open Access Numerical Results}

We consider only two tiers of limited interference for open access case. Fig. 6 previews the first tier edge user area spectral

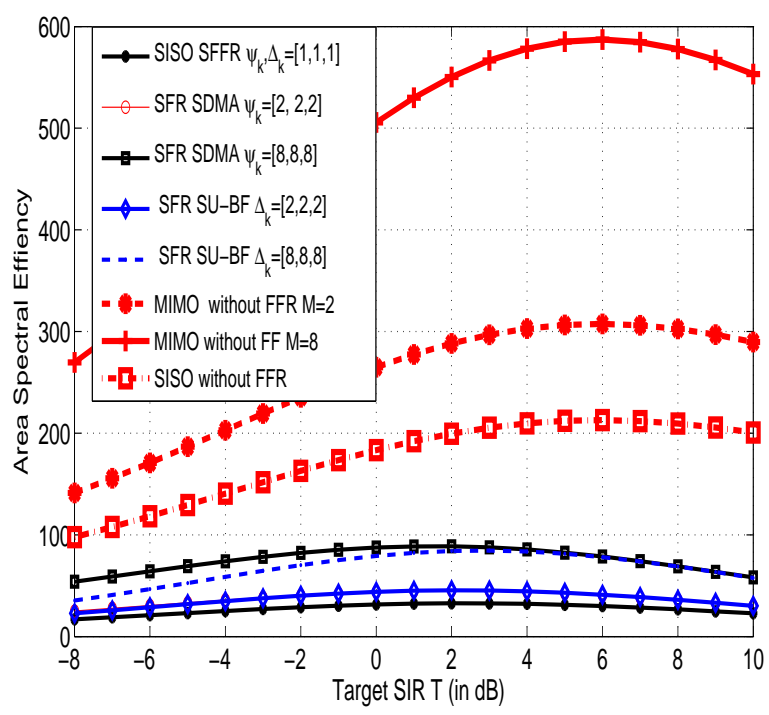

Fig. 5: Closed Access SFR DL ASE for first tier cell edge user at $\beta_{k}=[2,2,2]$ in HetNet.

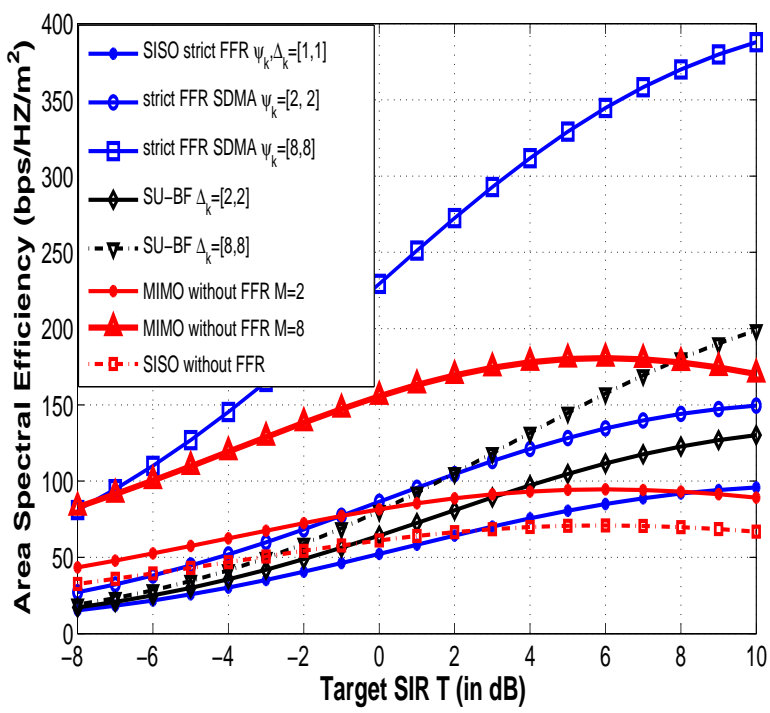

Fig. 6: Open Access Strict FFR DL ASE for first tier cell edge user in HetNet.

efficiency for SISO HetNet, MIMO HetNet with $M=2$, and 8 , and MIMO strict FFR for (SISO, SDMA, and SU-BF ). SDMA Strict FFR ASE outperforms other ASEs in case of open access. It is obvious that ASE curve of SDMA SFR with $\psi_{k}=[8,8]$ has the greatest ASE performance

Fig.7 shows the ASE curves in case of SFR, open access and at $\beta_{k}=[16,16]$. It is obvious from the figure that, at low target SIR values MU-MIMO dominates. However at higher ones, SFR SDMA dominates. Performance of SU-BF, and SDMA are nearly similar at low number of antennas, i.e. $M=1,2$. SISO SFR curve performance is lower than SISO HetNet because of the large interference resulted from using SFR. 


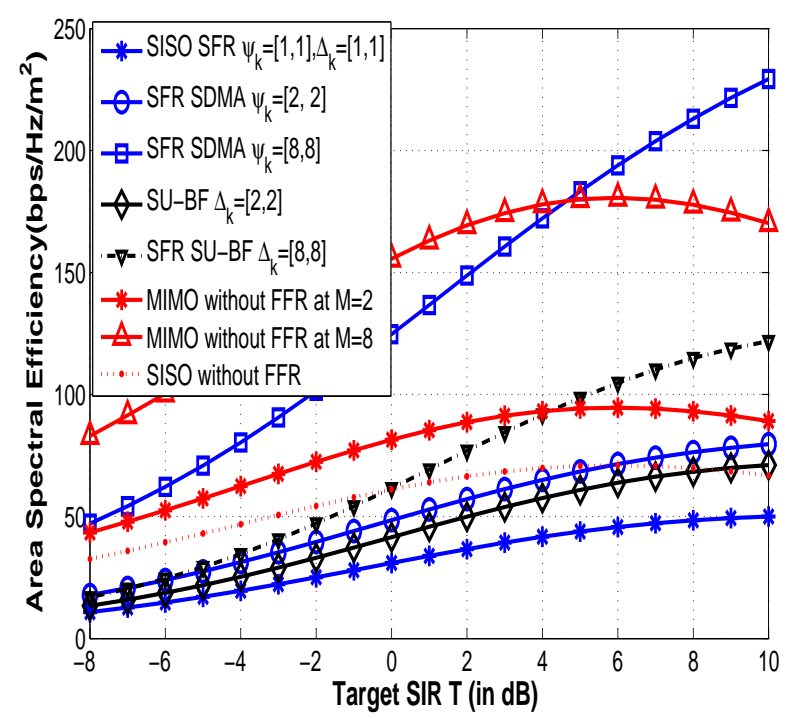

Fig. 7: Open Access SFR DL ASE for first tier cell edge user at $\beta_{k}=[16,16,16]$ in HetNet.

\section{CONCLUSION}

This paper compares SISO, MU-MIMO, strict FFR MIMO HetNets, closed FFR MIMO HetNets in terms of their ASE. We utilized PPP to model the BSs locations because of the their random deployment. ASEs are evaluated based on coverage probability functions for Strict FFR and Soft Frequency Reuse (SFR) in MIMO HetNets for both closed and open access cases. ASE is important for the systems that serve a large number of users, as it considers them. Numerical results showed that strict FFR, SDMA case has large ASE at high target threshold values, however at lower ones MU-MIMO ASE dominates. For better ASE MIMO HetNet system it is recommended to use adaptive technique between strict FFR MIMO and MU-MIMO in terms of target threshold SIR. SFR MIMO HetNet ASE is lower than MU-MIMO because of the large interference resulted from using SFR partitioning technique. Generally MIMO HetNets have large ASE than SISO HetNets.

\section{ACKNOWLEDGMENT}

This research is funded by the Ministry of Higher Education (MoHE) of Egypt through Ph.D fellowship. Our sincere thanks to EJUST University,Egypt and EJUST center, Kyushu University, Japan for their guidance, support, and encouragement. Also, The authors would like to thank ENG. He Zhuang, Keio University, Japan for his great support, and help.

\section{REFERENCES}

[1] J. G.Andrews, F. Baccelli, and R.K. Ganti, A Tractable approach to coverage and rate in cellular networks, IEEE Trans.on Communications, vol.59, no.11, pp.3122-3134, Nov.2011.

[2] G.Boudreau, J.Panicker, N.Guo, R.Chang, N.Wang, and S.Vrzic, "Interference Coordination and Cacellation for $4 G$ Networks,'IEEE Commun. Mag., vol 48, no.8, pp.7481, April 2009.

[3] M.Strend, T. Ottosson, A. Ahlen, and A. Svensson, "Attaining both coverage and high spectral effiency with adaptive OFDM down-links Proc. VTC 2003 Fall, vol. 4, pp.2486-2490, Oct. 2003.
[4] K. Doppler, C. Wijting, and K. Valkealahti,"Interference aware scheduling for soft frequency reuse, in Proc. 2009 IEEE Veh. Technol. Conf. Spring, pp.1-5, 2009.

[5] T. D.Novlan, R. K.Ganti, A. Ghosh, and J. G.Andrews,“ Analytical Evaluation of Fractional Frequency Reuse for OFDMA Cellular Networks, IEEE Trans. on wireless communications, vol.10, pp.4294-4305,Dec. 2011.

[6] T. Novlan, J. G. Andrews, I. Sohn, R. K. Ganti, and A.Ghosh,"Comparison of Fractional Frequency Reuse Approaches in the OFDMA cellular DL, Global Telecommunications Conference (GLOBECOM 2010), pp.1-5, 6-10 Dec. 2010.

[7] 3GPP TSG RAN WG1 no 42 R1-050764, Inter-cell interference handling for E-UTRA. 3GPP TSG RAN WG1 no 42 Rl-050764, 2005.

[8] "SFR.soft frequency reuse scheme for UTRAN LTE $3 G P P$.Huwaei,R1-050507,may 2005.

[9] S. M. Hashima, S. Elnoubi, M. Alghoniemy, H. Shalaby, O. Muta, and I. Mahmoud,"Analysis of Frequency Reuse Cellular systems Using worst Case Signal to Interference Ratio, "12 ${ }^{\text {th }}$ IEEE ConTel Conf, Croatia, 26-28 June 2013.

[10] S. M. Hashima, S. Elnoubi, M. Alghoniemy, H. Shalaby, O. Muta, and H.Furukawa," Performance Analysis of Frequency Reuse based on Using worst Case Signal to Interference Ratio in OFDMA Downlink Systems," Personal Indoor and Mobile Radio Communications (PIMRC), 2013 IEEE 24th International Symposium on , pp.616-620, 8-11 Sept. 2013.

[11] T. D. Novlan, R. K. Ganti, A. Ghosh, and J. G. Andrews, “ Analytical Evaluation of Fractional Frequency Reuse for Heterogeneous Cellular Networks, IEEE Trans. on communications, vol.60, no.7, pp.2029-2039 July. 2012.

[12] D. Stoyan, W. Kendall, and J. Mecke,"Stochastic Geometry and its applications, 2nd edition, John Wiley and sons, 1996.

[13] D. B. Taylor, H. S. Dhillon, T. D. Novlan, J. G. Andrews," Pairwise interaction processes for modeling cellular network topology, Global Communications Conference (GLOBECOM), 2012 IEEE , pp.4524:4529, 3-7 Dec. 2012.

[14] B. Blaszczyszyn, M. k. karry, and H. P. Keeler,“Using poisson processes to model lattice cellular networks, avaliable online:arxiv.org/abs/1207.7208.

[15] H. Huang, C. B. Papadias, and S. Venkatesan,“ MIMO Communication for Celllar Networks, Springer, 2012.

[16] H. S. Dhillon, R. K.Ganti, F. Baccelli, J. G.Andrews,“ Modeling and Analysis of K-Tier Downlink Heterogeneous Cellular Networks, Selected Areas in Communications, IEEE Journal on, vol.30, no.3, pp.550-560, April. 2012.

[17] H.S. Jo, Y. J. Sang, P. Xia, J.G.Andrews, "Heterogeneous Cellular Networks with Flexible Cell Association: A Comprehensive Downlink SINR Analysis, IEEE Transactions on Wireless Communications, vol.11, no.10, pp.3484-3495, October 2012

[18] S. Mukherjee,"Distribution of Downlink SINR in Heterogeneous Cellular Networks, IEEE Journal on Selected Areas in Communications, vol.30, no.3, pp.575-585, April 2012.

[19] R. W. Heath, and M. Kountouris, "Modeling heterogeneous network interference, Information Theory and Applications Workshop (ITA), 2012, pp.17-22, 5-10 Feb. 2012.

[20] H. S. Dhillon, M. Kountouris, and J. G. Andrews,"Downlink coverage probability in MIMO HetNets,2012 Conference Record of the Forty Sixth Asilomar Conference on Signals, Systems and Computers (ASILOMAR), pp. 683-687, 4-7 Nov. 2012.

[21] H. Zhuang, and T. Ohtsuki, “ Downlink coverage probability of Fractional Frequency Reuse for MIMO Heterogeneous Networks. IEICE Technical Report, online: http://www.ieice.org/ken/paper/20131018jBgD/eng/. 